\title{
Satellite infall and mass deposition on the Galactic center
}

\author{
S. C. Gallego and J. Cuadra \\ Instituto de Astrofísica, Pontificia Universidad Católica de Chile \\ email: sgallego@astro.puc.cl; jcuadra@astro.puc.cl
}

\begin{abstract}
We modeled numerically the infall of a small satellite galaxy on to the inner 200 parsec of our Galaxy, to test whether such an event perturbs gas orbiting in the central molecular zone (CMZ), as recently proposed by Lang et al. (2013). This process could have driven a large gas inflow around $10 \mathrm{Myr}$ ago, explaining the past high accretion rate onto the supermassive black hole, and the presence of young stars in the inner parsecs of the Galaxy. Our simulations show a very small inflow of gas, not sufficient to produce the aforementioned effects.
\end{abstract}

Keywords. Galaxy: center - Galaxy: kinematics and dynamics

\section{Introduction}

Sgr A*, the Galactic center (GC) supermassive black hole, is characterized by its current very low luminosity (Genzel et al. 2010). However, the recent discovery of the "Fermi bubbles"(Dobler et al. 2010; Su et al. 2010) plus the already known presence of young stellar discs in the inner parsec (Paumard et al. 2006) suggest that accretion onto the black hole was much stronger in the past. Indeed, Zubovas \& Nayakshin (2012) show that the deposition of $\mathrm{a} \sim 10^{5} \mathrm{M}_{\odot}$ giant molecular cloud into the inner parsec $\sim 6 \mathrm{Myr}$ ago could explain the unusual birth of stars and the "Fermi bubbles".

According to Lang et al. (2013), a satellite galaxy infall through the central molecular zone (CMZ) region could perturb previously stable gas, driving it into the inner region. They estimated a deposition of $\sim 10^{6} \mathrm{M}_{\odot}$ of gas in a timescale of about $10 \mathrm{Myr}$, for a $\sim 10^{5} \mathrm{M}_{\odot}$ satellite. This event would not only explain the nuclear activity, but also the formation of other young stellar clusters, the Arches and the Quintuplet, in the inner 30 pc.

Here we present preliminary results of our numerical study to test this scenario, which will be completed by Gallego \& Cuadra (in prep).

\section{Physical model and numerical set-up}

We model the satellite infall scenario with SPH simulations using the public Gadget2 code (Springel 2005). We include the gas of the CMZ as SPH particles, the satellite galaxy as a single dark matter particle, and the effect of the rest of the Galaxy is included as an external potential. Centrifugal and Coriolis forces were introduced in the code as we are working in the reference system of the bar.

The Galaxy potential is taken from Zhao et al. (1994),

$$
\Phi(r, \theta, \phi)=4 \pi G \rho_{0} r_{0}^{2}\left(\frac{r}{r_{0}}\right)^{\alpha} P(\theta, \phi),
$$

where $P(\theta, \phi)$ is a combination of associated Legendre functions and sets the degree of 


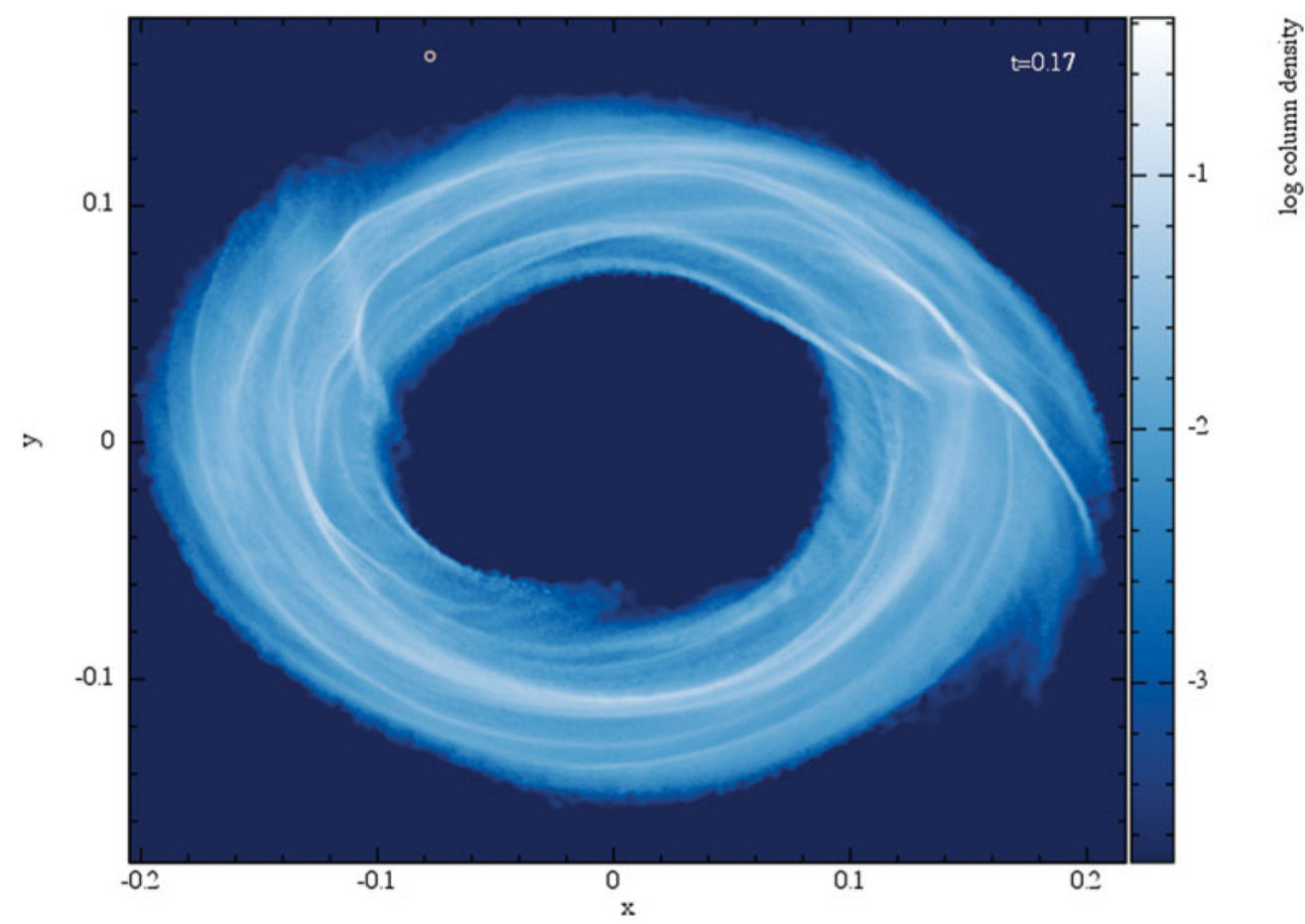

Figure 1. Column density map of the gas in the fiducial simulation at $166 \mathrm{Myr}$. The white circle corresponds to the satellite position. The CMZ gas is perturbed by the satellite, but mostly remains within its original radial range, producing little inflow. [A COLOR VERSION IS AVAILABLE ONLINE.]

oblateness/prolateness and non-axisymmetry. For details and the numerical values of the parameters, see Kim et al. (2011).

The CMZ follows the density distribution of Ferrière et al. (2007), truncated between 130 and $230 \mathrm{pc}$. The initial conditions, periodic closed orbits in the potential above, were obtained with the shooting technique.

Our fiducial simulation uses 300,000 particles for the ring, with a total mass of $10^{7} \mathrm{M}_{\odot}$ and a fixed temperature of $100 \mathrm{~K}$, plus a satellite with a size of $10 \mathrm{pc}$ and a mass of $10^{6} \mathrm{M}_{\odot}$, in an orbit that crosses several times the CMZ.

\section{Results and conclusion}

In the simulation, only a small fraction of the gas $\left(\sim 10^{3} \mathrm{M}_{\odot}\right)$ reaches the inner $80 \mathrm{pc}$ $60 \mathrm{Myr}$ after the satellite collision, and a completely negligible amount reaches the interesting inner scales $(10-30 \mathrm{pc})$. Other simulations with different parameters for the system do not change this result. In conclusion, the satellite perturbation does not seem sufficient to produce neither star clusters at $30 \mathrm{pc}$, nor nuclear activity.

This research was partially funded by CONICYT-Chile through FONDECYT (11100240), Basal (PFB0609) and Anillo (ACT1101) grants. We thank the IAU for travel grants to attend this meeting.

\section{References}

Genzel R., Eisenhauer F., \& Gillessen S. 2010, Rev. Mod. Phys. 82, 3121

Dobler G., Finkbeiner D. P., Cholis I., Slatyer T., \& Weiner N. 2010, ApJ 717, 825 
Ferrière, K., Gillard, W., \& Jean, P. 2007, A\& A 467, 611627

Kim, S. S., Saitoh, T. R., Jeon, M., Figer, D., Merritt, D., \& Wada, K. 2011, ApJ 735, L11

Lang, M., Holley-Bockelmann, K., Bogdanovic, T., et al. 2013, MNRAS 430, 2574

Paumard, T., et al. 2006, ApJ 643, 1011

Paumard, T., et al. 2006, MNRAS 364, 1005

Su, M., Slatyer, T. R., \& Finkbeiner, D. P. 2010, ApJ 724, 1044

Zhao, H., Spergel, D., \& Rich, R. 1994, A\&A 513, A35

Zubovas, K. \& Nayakshin, S. 2012, MNRAS 424, 666 